\title{
Socioeconomic Status Is Related to Pubertal Development in a German Cohort
}

\author{
Lea Oelkers $^{a} \quad$ Mandy Vogel $^{a} \quad$ Agnes Kalenda $^{a}, b \quad$ Hans Christian Surup ${ }^{a}$ \\ Antje Körner $^{a, b}$ Jürgen Kratzsch ${ }^{c}$ Wieland Kiess ${ }^{a, b}$ \\ aLIFE - Leipzig Research Center for Civilization Diseases, Leipzig University, Leipzig, Germany; bepartment \\ of Woman and Child Health, Hospital for Children and Adolescents and Center for Pediatric Research Leipzig \\ $(\mathrm{CPL})$, Leipzig University, Leipzig, Germany; ' Institute of Laboratory Medicine, Clinical Chemistry and Molecular \\ Diagnostics (ILM), Leipzig University, Leipzig, Germany
}

\section{Keywords}

Puberty · BMI - Luteinizing hormone · Follicle-stimulating hormone - Socioeconomic status · Gonadarche · Thelarche · Menarche $\cdot$ Voice mutation

\begin{abstract}
Introduction: Current health literature suggests that there has been a decline in the age of pubertal onset and that pubertal onset/duration of puberty may, besides weight status, be influenced by socioeconomic context. Objective: The goal of this study was to determine whether pubertal onset/ duration and puberty-triggering hormones luteinizing hormone ( $\mathrm{LH}$ ) and follicle-stimulating hormone (FSH) vary according to socioeconomic status (SES). Moreover, we aimed to propose cutoff values of serum LH and FSH for predicting gonadarche in boys. Methods: 2,657 apparently healthy children and adolescents between 5.5 and 18 years from the area of Leipzig were recruited from the LIFE Child study. Age at pubertal onset/end of puberty was given in 738/573 children, respectively. Anthropometric parameters of puberty, blood measurements of $\mathrm{LH}$ and $\mathrm{FSH}$, and questionnaires assessing SES were evaluated. Results: Lower SES was associated with earlier thelarche and longer duration of puberty in overweight/obese girls, whereas age of menarche was not
\end{abstract}

karger@karger.com www.karger.com/hrp

Karger $\stackrel{\text { ' }}{5}$

GOPEN ACCESS
(C) 2021 The Author(s)

Published by S. Karger AG, Basel

This is an Open Access article licensed under the Creative Commons Attribution-NonCommercial-4.0 International License (CC BY-NC) (http://www.karger.com/Services/OpenAccessLicense), applicable to the online version of the article only. Usage and distribution for commercial purposes requires written permission. affected. In boys with low SES, a trend versus earlier puberty onset can be seen. Lower SES was significantly associated with boys' age at mutation. No significant differences in boys' and girls' serum levels of LH and FSH during puberty according to SES were observed. Serum LH levels of $0.56 \mathrm{IU} / \mathrm{L}$ and serum FSH levels of $1.74 \mathrm{IU} / \mathrm{L}$ showed the best prediction of gonadarche in boys. Conclusion: Puberty onset/duration and boys' age at mutation is affected by SES. The proposed cutoff levels for serum LH and FSH could provide a serological tool to determine gonadarche in boys.

\section{(C) 2021 The Author(s)}

Published by S. Karger AG, Basel

\section{Introduction}

For a better understanding of children's and adolescents' sexual and reproductive health, it is important to have access to up-to-date, region-specific data on pubertal timing. Puberty as a critical time of growth and development is influenced by a range of genetic, environmental, and lifestyle factors $[1,2]$. Variation in pubertal timing may affect health risks. Previous studies showed that early puberty [3] or early menarche $[4,5]$ might lead to adverse outcomes such as increased substance use [3], earlier sexual activity [4], and increased risk of breast can- 
Fig. 1. Process of case selection.



cer [5]. In addition, late maturity among boys has been associated with behavior problems [6] and lower educational attainment [7]. For quite some time, research discusses the association of elevated BMI with earlier puberty [8]. Additionally, growing scientific evidence points to an association of children's socioeconomic status (SES) with a wide array of health-related, cognitive, and socioemotional outcomes [9-11]. The German Health Survey for Children and Adolescents (KiGGS) showed that children and adolescents with low SES are half as likely to have good general health as those with high SES [11]. We could recently demonstrate a strong association between SES and child health and development [9]. A low SES was, for example, associated with emotional disorders, behavioral problems, a less healthy lifestyle, and less physical activity $[9,12,13]$. Despite the strong link between SES and different aspects of child health, research results are inconclusive with regard to the influence of SES on pubertal timing. An Australian cohort study showed that exposure to socioeconomic disadvantage predicts earlier puberty onset in both girls and boys [14] and a Canadian study found that adolescents with high SES were less likely to have entered puberty by the age of 13 [2]. Other studies from the USA and Germany showed that lower SES is associated with earlier menarche $[11,15,16]$. However, a recent meta-analysis, that also included studies from less developed countries, did not find a significant association between low SES and early pubertal timing [17]. So far, little research activity has focused on associations between SES and the duration of puberty.

Our study provides current data on critical steps during pubertal development in healthy adolescents from the German LIFE Child study cohort. Moreover, we investigated whether there is an association between SES and pubertal onset/duration as well as age at menarche and mutation. Finally, we investigated whether SES relates to serum values of the puberty-triggering hormones luteinizing hormone (LH) and follicle-stimulating hormone (FSH) and propose a cutoff value of serum $\mathrm{LH}$ and FSH for predicting gonadarche in boys.

\section{Methods}

\section{Study Population}

Our study originates from the LIFE Child Study, which is a part of the Leipzig Research Centre for Civilization Diseases (LIFE). LIFE Child aims at a better understanding of the etiology of lifestyle diseases. It collects detailed longitudinal and cross-sectional information from children and adolescents with mostly Caucasian ethnic background aged 0-20 years as well as from their parents. Precise study characteristics are described elsewhere [18]. For the present study, data from children and adolescents between 5.5 and 18 years with annual visits from 2011 to 2019 were analyzed. After applying the exclusion criteria (Fig. 1), the sample consisted of 7,986 visits from 2,657 children. 
Table 1. Demographic characteristics of the LIFE child study cohort

\begin{tabular}{|c|c|c|}
\hline & Female & Male \\
\hline Age range, yrs & $5.50-18.0$ & $5.50-18.0$ \\
\hline Number of included cases & $n=1,295$ & $n=1,362$ \\
\hline Number of included visits & $n_{\mathrm{vis}}=3,853\left(48.7 \%^{1}\right)$ & $n_{\text {vis }}=4,133\left(51.3 \%^{1}\right)$ \\
\hline \multicolumn{3}{|c|}{ Distribution of SES in study population ${ }^{2}$} \\
\hline$n$ (percent) & $160(13.2)$ & $145(11.3)$ \\
\hline $\mathrm{BMI}^{\mathrm{SDDS}}{ }^{3}$ & $1.06 \pm 1.51$ & $0.92 \pm 1.47$ \\
\hline \multicolumn{3}{|l|}{ Mid-SES } \\
\hline$n$ (percent) & $712(58.6)$ & $740(57.8)$ \\
\hline $\mathrm{BMI}^{\mathrm{S}} \mathrm{SDS}^{3}$ & $0.15 \pm 1.19$ & $0.19 \pm 1.18$ \\
\hline \multicolumn{3}{|l|}{ High SES } \\
\hline$n$ (percent) & $344(28.2)$ & $395(30.9)$ \\
\hline${\mathrm{BMI}-\mathrm{SDS}^{3}}^{3}$ & $-0.05 \pm 0.95$ & $-0.08 \pm 0.92$ \\
\hline \multicolumn{3}{|l|}{ Visits per pubertal stage, $\mathrm{n}$} \\
\hline \multicolumn{3}{|l|}{ Pubertal stage, $n_{\text {vis }}$} \\
\hline 1 & 1,516 & 1,647 \\
\hline 2 & 473 & 462 \\
\hline 3 & 394 & 184 \\
\hline 4 & 392 & 247 \\
\hline 5 & 675 & 270 \\
\hline Menarche & $n=576$ & \\
\hline Mutation & & $n=452$ \\
\hline
\end{tabular}

SES, socioeconomic status; SD, standard deviation. ${ }^{1} 48,7 \%$ of the visits included were female, $51.3 \%$ were male. ${ }^{2}$ SES and BMI-SDS at baseline visit. ${ }^{3}$ Values expressed as mean \pm SD.

\section{Anthropometric Indicators of Puberty and BMI}

The pubertal stage was assessed for both sexes according to Tanner $[19,20]$ by well trained, qualified personnel. The breast developmental stage (B) in girls was evaluated by palpation of glandular breast. The median age at onset of puberty was estimated as the age at which $50 \%$ of the study subjects entered pubertal stage 2 (defined as the age when both of the following conditions meet: (a) for males testicular volume $>3 \mathrm{~mL}$ [21] and pubic hair stage (PH) 1 or 2 and (b) for females B2 and PH1-3). Therefore, pubertal onset in girls was mainly defined by the onset of thelarche. Pubertal stage 5 was considered as mature and defined both as a testicular volume $\geq 7 \mathrm{~mL}$ and $\mathrm{PH} 5$ or PH6 in boys and both $\mathrm{B} 5$ and PH4-6 in girls. Median end of puberty was estimated as the age at which $50 \%$ of the study subjects entered pubertal stage 5 . Because too few subjects had both a known entry and end of puberty, the duration of puberty was defined by the time interval from the median pubertal onset to the median end of puberty in each subgroup. Age at menarche was obtained from 576 girls. 452 boys provided information about their age at mutation (voice change). Children's height, weight, and BMI-SDS were assessed as described before [18]. All subjects were assigned to 1 of 2 weight groups according to the criteria of the German Obesity Task Force Group [22]. BMISDS from -1.28 to +1.28 was defined as normal weight (NW) and above +1.28 as overweight or obese (OW). Children with a BMISDS below -1.28 were excluded from analyses.

\section{Socioeconomic Status}

The SES of children was assessed at their first visit recorded in the data using the "Winkler-Stolzenberg-Index" [9, 12, 13, 23].
This index comprises 3 scales measuring parental education, occupational status and household equivalized disposable income (for more information see [23]). In the case of missing values in 1 of the 3 scales, it was substituted by the mean of the remaining 2 indicators. The resulting scale ranges from 3 to 21 points. Based on the score, 3 categories of SES were defined: low (3-8 points), mid (9-14 points), and high (15-21 points).

\section{Laboratory Measures}

Blood samples were collected between 7.30 and 10.30 a.m. in a fasting state. Samples were sent to the Institute of Laboratory Medicine, University Hospital of Leipzig, for analysis on the same day. $\mathrm{LH}$ and FSH were analyzed by electrochemiluminescence immunoassay (Roche, Mannheim, Germany). The assay was performed according to the specification of the manufacturer. The detection limit was $0.1 \mathrm{IU} / \mathrm{L}$. For LH, intra-assay coefficients of variation $(\mathrm{CV})$ were $\leq 1.2 \%$ and inter-assay CVs were $\leq 2.2 \%$ at the concentration range of $6.2-164 \mathrm{IU} / \mathrm{L}$. For FSH, intra-assay CVs were $\leq 2.8 \%$ and inter-assay $\mathrm{CVs}$ were $\leq 4.5 \%$ at the concentration range of $6.0-178 \mathrm{IU} / \mathrm{L}$.

\section{Statistics}

BMI was transformed into age- and sex-adapted standard deviation scores (SDS) according to the German standards [22]. For determining puberty onset, hierarchical logistic regression analyses were conducted stratified by sex. To account for multiple measurements per person, hierarchical models were applied, adding the subject as a random intercept. LH and FSH levels were compared between the different pubertal stages applying an ANOVA 
Table 2. Parameters of pubertal development by gender, weight group 2 and 3 , and SES

\begin{tabular}{|c|c|c|c|c|c|c|c|c|}
\hline & \multicolumn{4}{|l|}{ Female } & \multicolumn{4}{|l|}{ Male } \\
\hline & $\begin{array}{l}\text { age at } \\
\text { pubertal onset, } \\
\operatorname{yr}(n)\end{array}$ & $\begin{array}{l}\text { age at end } \\
\text { of puberty, } \\
\text { yr }(n)\end{array}$ & $\begin{array}{l}\text { duration } \\
\text { of puberty, } \\
\text { yr }\end{array}$ & $\begin{array}{l}\text { age at } \\
\text { menarche, } \\
\text { yr }(n)\end{array}$ & $\begin{array}{l}\text { age at } \\
\text { pubertal onset, } \\
\operatorname{yr}(n)\end{array}$ & $\begin{array}{l}\text { age at end } \\
\text { of puberty, } \\
\text { yr }(n)\end{array}$ & $\begin{array}{l}\text { duration } \\
\text { of puberty, } \\
\text { yr }\end{array}$ & $\begin{array}{l}\text { age at } \\
\text { mutation, } \\
\operatorname{yr}(n)\end{array}$ \\
\hline All & $10.18(372)$ & $14.77(379)$ & 4.59 & $12.55(576)$ & $11.01(366)$ & $15.24(194)$ & 4.23 & $13.26(452)$ \\
\hline $\mathrm{NW}^{2}$ & $10.3(301)$ & $14.99(295)$ & 4.69 & $12.6(452)$ & $11.02(301)$ & $15.27(154)$ & 4.25 & $13.23(374)$ \\
\hline High SES & $10.38(80)$ & $15.14(58)$ & 4.76 & $12.85(100)$ & $11.17(102)$ & $15.14(57)$ & 4.17 & $13.15(117)$ \\
\hline $\mathrm{OW}^{3}$ & $9.51(74)$ & $13.94(99)$ & 4.43 & $12.22(152)$ & $10.95(65)$ & $15.12(45)$ & 4.17 & $13.49(101)$ \\
\hline Low SES & $8.97(25)$ & $13.88(29)$ & 4.91 & $12.35(39)$ & $10.97(15)$ & $14.76(10)$ & 3.78 & $13.13(18)$ \\
\hline Mid-SES & $9.65(35)$ & $14.05(49)$ & 4.4 & $12.24(81)$ & $10.92(37)$ & $15.10(28)$ & 4.18 & $13.10(65)$ \\
\hline High SES & $10.02(10)$ & $13.78(14)$ & 3.76 & $12.31(22)$ & $11.24(9)$ & $15.41(5)$ & 4.17 & $14.29(15)$ \\
\hline
\end{tabular}

Age is expressed as median. Importantly, lower SES values in OW girls were associated with earlier puberty onset and longer duration of puberty and in NW/OW boys with a trend toward earlier puberty onset and with higher/lower age at mutation. NW, normal weight; OW, overweight or obese; SES, socioeconomic status. ${ }^{1}$ As defined by the time interval from the median pubertal onset to the median end of puberty in each subgroup. ${ }^{2} \mathrm{NW}=$ normal weight, as defined by BMI-SDS from -1.88 to $1.28 .{ }^{3}$ OW $=$ overweight or obese, as defined by BMI-SDS $>1.28$.

followed by the Tukey Honest Significant Difference test. LH- and FSH-cutoffs for puberty onset were obtained using ROC analysis. All statistical analyses were performed with the statistical software R V.3.4.0.

\section{Results}

\section{Study Characteristics}

2,657 children ( $48.7 \%$ female) with a total of 7,986 visits were included. Demographic background characteristics of the study sample are presented in Table 1. A low SES was less frequently observed than a mid or a high SES. Children with low SES were more likely to be OW compared to children with middle or high SES.

\section{Anthropometric Parameters of Pubertal Development}

Puberty started earlier and lasted longer in girls than in boys (Table 2). The median age of pubertal onset was 10.18 years in girls and 11.01 years in boys. The median age when reaching pubertal stage 5 was 14.77 years in girls and 15.24 years in boys. The duration of puberty was 4.59 years in girls and 4.23 years in boys. In girls, the median age at menarche was 12.6 years. In boys, the median age of voice break was 13.26 years. In girls, higher BMI values were significantly associated with an earlier pubertal onset (NW: 10.3 years vs. OW: 9.51 years, $p<0.05$ ) and an earlier age at menarche (NW: 12.6 years vs. OW: 12.22 years, $p<0.05)$. In boys, however, pubertal onset was not significantly associated with weight group. Lower SES values were associated with earlier puberty in OW girls (Fig. 2). Girls with low SES entered puberty at a median age of 8.97 years, whereas girls with mid- and high SES did not enter puberty until the age of 9.65 and 10.02 years, respectively. In other terms, at the age of 8.97 years, $50 \%$ of the girls with low SES had already entered puberty compared to $23 \%$ of the girls with mid-SES $(p=0.17)$ and $3.8 \%$ of the girls with high SES $(p=0.05)$. Furthermore, in OW girls with low SES, the duration of puberty was 1.15 years longer than in girls with high SES (4.91 vs. 3.76 years). In both NW and OW boys with low SES, a trend toward earlier puberty was observed but did not reach statistical significance. The age of pubertal onset in NW boys was 10.73 and 11.17 years for low and high SES ( $p=$ 0.13 ), the age of pubertal onset in OW boys was 10.97 and 11.24 years for low and high SES $(p=0.21)$. In boys with low SES, the duration of puberty was slightly longer for OW boys than in NW boys ( 4.51 vs. 3.78 years). We found no significant difference in age at menarche between girls of low, mid-, and high SES, neither among NW nor among OW girls. However, age at mutation in boys was significantly associated with lower SES values: NW boys with low SES experienced mutation significantly later than boys with high SES (14.02 vs. 13.15 years, $p<0.05$ ), whereas OW boys with low SES experienced mutation significantly earlier than boys with high SES (13.13 vs. 




Fig. 2. The probability of puberty onset at each age according to gender (a, male; b, female), weight group ${ }^{1,2}$, and SES. Lower SES values were associated with earlier puberty in $\mathrm{OW}^{2}$ girls. ${ }^{1} \mathrm{NW}$ as defined by BMI-SDS from -1.88 to $1.28 .{ }^{2} \mathrm{OW}$ as defined by BMI-SDS $>1.28$. NW, normal weight; OW, overweight and obese; SES, socioeconomic status. 


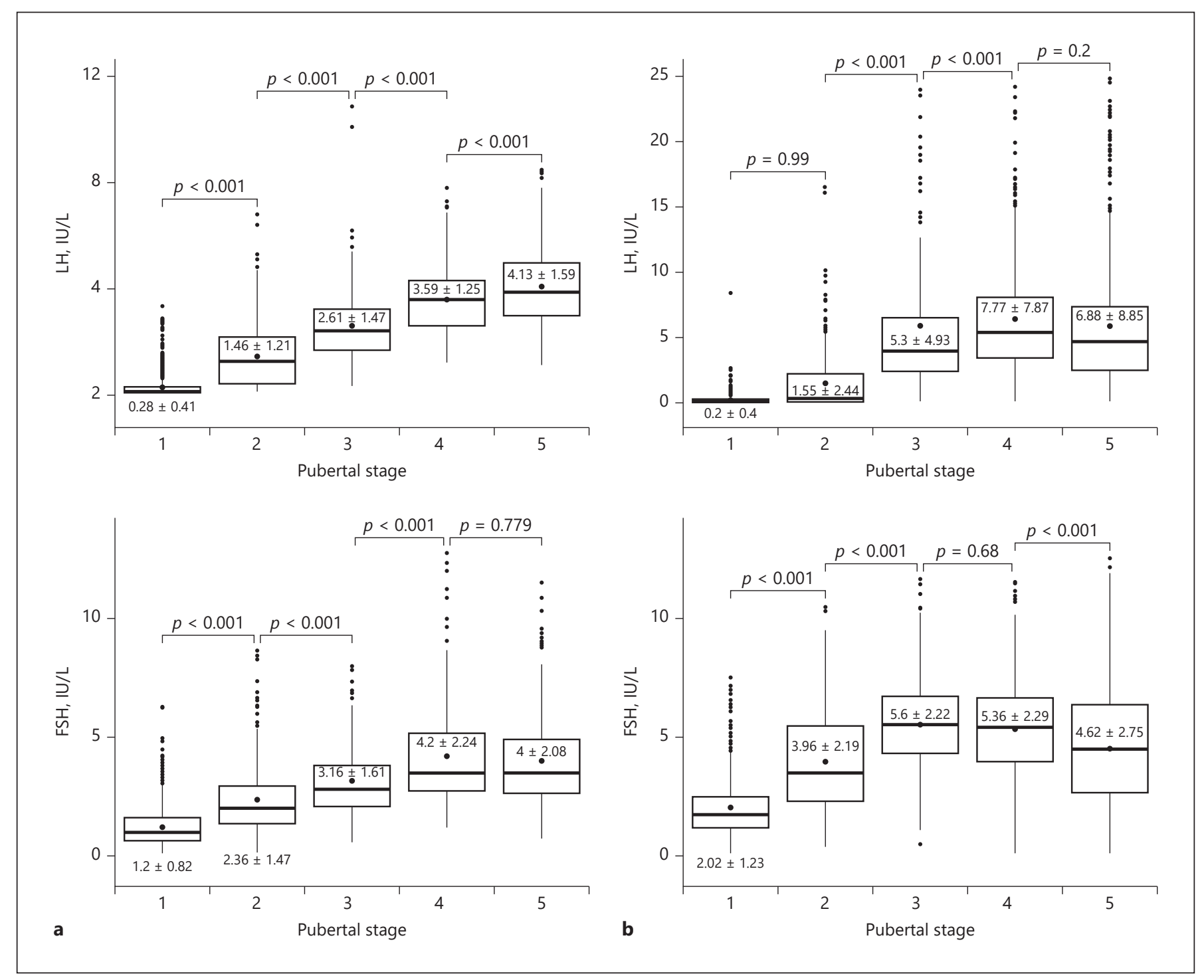

Fig. 3. LH and FSH serum values in boys (a) and girls (b) during puberty. The black dot within the boxplot represents the mean (additionally labeled with mean $\pm \mathrm{SD}$ ). LH, luteinizing hormone; FSH, follicle-stimulating hormone; SD, standard deviation.

14.29 years, $p<0.05)$. In summary, lower SES values were (a) associated with earlier and longer puberty in OW girls and (b) with a trend towards earlier puberty and with later/earlier age at mutation in NW/OW boys.

\section{Gonadotropin Measurements}

The mean and standard deviation of serum $\mathrm{LH}$ and FSH values in boys and girls across different stages of puberty are shown in Figure 3. In boys, mean serum LH levels increased significantly from pubertal stages $1-5$, mean serum levels of FSH changed significantly from pubertal stages 1-4 ( $p<0.001)$. Based on the ROC-curve, the best

Socioeconomic Status and Pubertal Timing cutoff value of serum $\mathrm{LH}$ for predicting gonadarche in boys was $0.56 \mathrm{IU} / \mathrm{L}$ with a sensitivity and specificity of 86 and $88 \%$, respectively (Fig. 4). The area under the curve was 0.906 (CI: $0.892-0.921$ ). The cutoff value of serum FSH was $1.74 \mathrm{IU} / \mathrm{L}$ with a sensitivity and specificity of $76 \%$. The area under the curve was 0.841 (CI: 0.823$0.859)$. In girls, there was no significant change in LH levels between pubertal stages 1 and 2. An increase can only be seen from stages 2-4. Although serum levels of FSH rise significantly from pubertal stages $1-3$, a possible cutoff for thelarche as determined by ROC analysis showed only poor sensitivity and specificity. Therefore, cutoff 


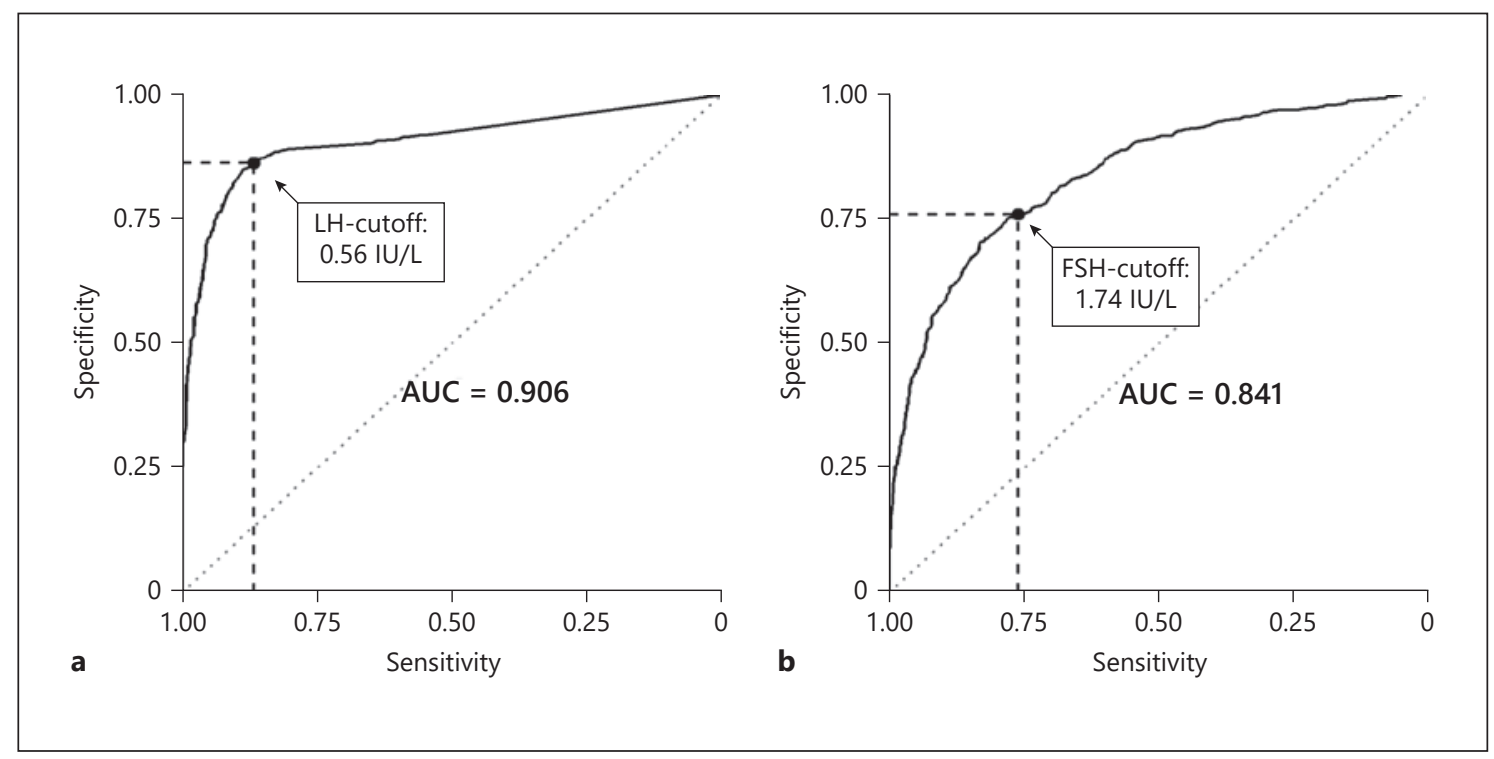

Fig. 4. Receiver operating characteristic curve of LH (a) and FSH (b) to predict gonadarche in boys as defined by testicular volume $\geq 3 \mathrm{~mL} . N=361$ (total number of boys entering gonadarche). LH-cutoff: 0.56 IU/L, AUC: 0.906 (0.892-0.921), sensitivity: 0.86, and specificity: 0.88. FSH-cutoff: $1.74 \mathrm{IU} / \mathrm{L}$, AUC: 0.841 (0.823-0.859), sensitivity: 0.76 , and specificity: 0.76 . LH, luteinizing hormone; FSH, follicle-stimulating hormone.

values of both LH and FSH seem inappropriate as serological markers for determining thelarche. No significant differences in girls' and boys' LH and FSH levels between the different SES groups were observed during puberty (results not shown).

\section{Discussion}

Pubertal Timing, BMI, and Socioeconomic Status in the Light of Existing Literature

Many countries are experiencing an epidemic of pediatric obesity, which is more prevalent in lower SES groups [24]. However, although it is well known that a critical body weight and/or fat mass must be achieved for the onset of puberty [25], the influence of BMI on pubertal development is still controversial [26-29]. In our study, elevated BMI was significantly associated with earlier thelarche in girls but not with earlier puberty in boys. This is in line with a large body of literature that supports the hypothesis that higher BMI is associated with earlier puberty in girls [28, 30-33]. However, a few controversial results in European cohort studies have also been published [26, 34]. In boys, the relationship between pubertal onset and BMI is less consistent $[8,11,28,29]$. Despite the possible influence of BMI on pubertal timing, BMI was not found to (solely) mediate the associations be- tween SES variables and pubertal onset [14], age at menarche [15], or breast development [24]. Our findings, too, suggest an effect of SES beyond the weight group effect. Especially, OW girls with low SES had earlier thelarche and longer puberty than OW girls with high SES. A Canadian study found girls from low SES enter puberty earlier and go through puberty at a slower pace [2]. A study from Australia, too, found an association of socioeconomic disadvantage and early puberty [14], whereas a recent meta-analysis did not find a significant association [17]. Regarding menarchal age, we found no associations with SES contrary to the German KiGGs study and studies from the US reporting a positive correlation [11, 15, 16]. In boys with low SES, we observed a trend of earlier puberty and an association of SES with age at mutation in both NW and OW boys. Interestingly, low SES was significantly associated with later mutation in NW boys, but, on the contrary, with earlier mutation in OW boys. However, these findings are based on self-reported data and should be viewed critically. Additionally, a comparison of pubertal timing between studies should be made carefully. Differences in design and composition of study cohorts, the methodology of measuring puberty onset and ongoing puberty as well as regional conditions differ widely and have to be seen in a geographical or ethnic context. 
Possible Links between Low Socioeconomic Status and Earlier Puberty

Pubertal timing may play a mediating role between early social disadvantages and health disparities later in life as suggested by Sun et al. [14]. The mechanisms by which socioeconomic disadvantage might contribute to the risk of early puberty are not yet clear and thus, speculative. We discussed the possible impact of BMI on early puberty. BMI was not found to exclusively mediate the associations between SES variables and pubertal timing. There may be other environmental, behavioral, and physiological effects by which SES influences early pubertal development. An alternative pathway by which SES might mediate puberty onset is through increased exposure to stress associated with lower SES [2, 13, 35-39]. Results from the German KiGGS study suggest that children and adolescents with low SES are half as likely as those with high SES to have good general health; they were significantly more affected by psychological and behavioral difficulties [11]. Exposures to stress could influence physiological and psychological processes, resulting, for example, in cell/molecular damage [36], poorer emotional health [12], and health behavior, like reduced physical activity and poor diet [13]. Moreover, exposure to early life stress could influence both the hypothalamic-pituitary-gonadal and hypothalamic-pituitary-adrenal axes and thus provide a link to advanced pubertal maturation [40]. Hochberg and Belsky [41] reviewed the understanding of pubertal maturation from an evolutionary life-history perspective. The life-history theory as a branch of evolutionary biology provides a theoretical framework for explaining the individual reproductive strategies that organisms use to allocate their limited time and energy to shaping the stages of their life. According to this theory, exposure to different childhood environments biases children toward different reproductive strategies that may include early pubertal timing $[14,37,42]$. The theory constitutes a set of assumptions. Belsky (1991) and Ellis (2004) proposed the psychosocial acceleration/developmental theory to account for the relationship between early pubertal maturation and environmental stressors, characterized by high levels of familial interpersonal stress and untrustworthy pair-bonds [37, 42]. Under such adverse conditions, girls show earlier and accelerated puberty because paternal investment is unreliable/not related to the reproductive success of offspring. Therefore, early puberty and a longer period of reproduction in children from disadvantaged contexts may also be seen as an advantage, though, according to Belsky (1991), probably more an advantage of quantity, less of quality [37]. On the

Socioeconomic Status and Pubertal

Timing metabolic level, the early stressful environment is supposed to lead to an internalization of problems, changes in metabolism and body fat and thus to an earlier onset of puberty $[14,37,42]$. Our study neither delivered data on early adverse life events nor biochemical markers of stress, and it gives no answer to the question of how this could influence centrally induced pubertal onset. Further research is needed to investigate the possibility of an evolutionary benefit of earlier puberty onset in case of a more demanding early environment.

\section{Luteinizing Hormone- and Follicle-Stimulating}

Hormone-Serum Levels as Markers for Puberty Onset

It is well known that $\mathrm{LH}$ and FSH as mediators of pubertal development increase with advancing puberty. To estimate a cutoff value for serum LH (0.56 IU/L) and serum FSH (1.74 IU/L) as serological predictors of gonadarche in boys, we compared these measures to testicular volume, a widely used anthropometric standard in the determination of puberty onset in boys [43]. An earlier Argentinian study found an optimal LH-cutoff of 0.65 IU/L and an FSH-cutoff of $2.5 \mathrm{IU} / \mathrm{L}$ in boys based on laboratory measures of 20 boys [44]. An Indian study found an optimal LH-cutoff value comparable to ours (0.53 IU/L, AUC: 0.59) [45]. The AUCs of the present study indicates that pubertal boys can be distinguished from prepubertal boys by determining LH and FSH with high sensitivity and specificity. The cutoff value could help to verify the puberty onset, though its validity is yet unclear. A single hormone measurement during clinical practice may not always predict pubertal onset among individual boys [46]. If verification of pubertal onset is needed, LH measurements following stimulation with gonadotropinreleasing hormone $(\mathrm{GnRH})$ or a gonadotropin-releasing hormone analog may be required [47]. In girls, LH and FSH show a larger variability across all puberty stages (as indicated in Fig. 3). It was, therefore, impossible to determine a cutoff level for puberty onset with good validity measures for either parameter.

\section{Strengths and Limitations}

Our study has limitations worth noting. We determined the duration of puberty based on median values of onset and end of puberty. Longitudinal data from a larger sample would allow for determination of individual puberty courses. Despite the large amount of study subjects, case numbers at least in some subgroups are small. We did not evaluate data on diurnal rhythm of LH and FSH serum values. As is true for most population-based studies, families with mid- and high SES are over-represented 
in our study sample. Personal initiative and interest in health issues are necessary prerequisites and probably less pronounced in people with lower SES [48]. Because our study cohort includes an unintended selection of presumably more health-conscious families, it is possible that the observed effects may be even more pronounced in the target population. Our study does not contain data on SES in early childhood. In some cases, the SES at first visit might not be equal to the SES in early life years crucially relevant for child development. The main strengths of our study are the large sample size and the standardized assessment that allowed us to provide a variety of pubertal parameters. To the best of our knowledge, this is the first European study that examines the impact of SES not only on pubertal turning points like menarche and puberty onset but on the duration of puberty. Cutoff values for $\mathrm{LH}$ and FSH in boys were determined with high diagnostic sensitivity and specificity. LIFE Child is 1 of the largest running cohorts of healthy children and adolescents in Europe and, therefore, well suited for the determination of representative pubertal and hormone parameters.

\section{Conclusion}

In summary, we established pubertal parameters including puberty onset and duration, age of menarche/ mutation, and pubertal hormone values of LH and FSH in a large sample of German children and adolescents. We showed that low SES is associated with earlier thelarche and longer puberty especially in OW girls and with a trend toward earlier puberty in boys. Finally, we propose cutoff values of serum LH and FSH to predict gonadarche in boys.

\section{Acknowledgements}

This publication was supported by LIFE - Leipzig Research Centre for Civilization Diseases, University of Leipzig. The authors are very grateful to the whole LIFE Child team and to all the families who participated in this study.

\section{Statement of Ethics}

Subjects (or their parents or guardians) have given their written informed consent to publish their case (including publication of images). All examination methods were in accordance with the principles of the Declaration of Helsinki and with the ethical standards of the national research committee. This study was approved by the Ethical Committee of the University of Leipzig (reference number: Reg. No. 264-10-19042010) and is registered under the trial number: NCT02550236.

\section{Conflict of Interest Statement}

There are no competing interests to declare.

\section{Funding Sources}

LIFE is funded by means of the European Union, by the European Regional Development Fund (ERDF) and by means of the Free State of Saxony within the framework of the excellence initiative of the Saxonian Ministry of Science and Arts (SMWK), Free State of Saxony, Germany.

\section{Author Contributions}

Wieland Kiess (W.K.) and Jürgen Kratzsch (J.K.) conceived the presented idea. Lea Oelkers (L.O.), Hans Surup, and Mandy Vogel (M.V.) developed the theory and performed the computations. M.V. verified the analytical methods. W.K., J.K., M.V., Agnes Kalenda, and Antje Körner discussed the results. L.O. drafted the manuscript, which was critically revised by all the authors.

\section{References}

1 Parent AS, Franssen D, Fudvoye J, Pinson A, Bourguignon JP. Current changes in pubertal timing: revised vision in relation with environmental factors including endocrine disruptors. Endocr Dev. 2016;29:174-84.

2 Arim RG, Shapka JD, Dahinten VS, Willms JD. Patterns and correlates of pubertal development in Canadian youth: effects of family context. Can J Public Health. 2007;98(2):91-6.

3 Patton GC, McMorris BJ, Toumbourou JW, Hemphill SA, Donath S, Catalano RF. Puberty and the onset of substance use and abuse. Pediatrics. 2004;114(3):e300-6.

4 Kaltiala-Heino R, Rimpelä M, Rissanen A, Rantanen P. Early puberty and early sexual activity are associated with bulimic-type eat- ing pathology in middle adolescence. J Adolesc Health. 2001;28(4):346-52.

5 Velie EM, Nechuta S, Osuch JR. Lifetime reproductive and anthropometric risk factors for breast cancer in postmenopausal women. Breast Dis. 2005;24:17-35.

6 Graber JA, SeeleyJR, Brooks-Gunn J, Lewinsohn $\mathrm{PM}$. Is pubertal timing associated with psychopathology in young adulthood. J Am Acad Child Adolesc Psychiatry. 2004;43(6):718-26.

7 Koivusilta L, Rimpelä A. Pubertal timing and educational careers: a longitudinal study. Ann Hum Biol. 2004;31(4):446-65.

8 Biro FM, Kiess W. Contemporary trends in onset and completion of puberty, gain in height and adiposity. Endocr Dev. 2016;29:122-33.
9 Poulain T, Vogel M, Sobek C, Hilbert A, Körner A, Kiess W. Associations between socio-economic status and child health: findings of a large German cohort study. Int J Environ Res Public Health. 2019 Feb 26;16(5): 677.

10 Bradley RH, Corwyn RF. Socioeconomic status and child development. Annu Rev Psychol. 2002;53:371-99.

11 Lampert T, Kurth BM. Sozialer Status und Gesundheit von Kindern und Jugendlichen: Ergebnisse des Kinder- und Jugendgesundheitssurveys (KiGGS): Socioeconomic status and health in children and adolescents: Epidemiologie und Gesundheitsberichterstattung. 2016 Jan 1. 
12 Herrmann J, Vogel M, Pietzner D, Kroll E, Wagner O, Schwarz S, et al. Factors associated with the emotional health of children: high family income as a protective factor. Eur Child Adolesc Psychiatry. 2018;27(3):319-28.

13 Rieger K, Vogel M, Engel C, Ceglarek U, Harms K, Wurst U, et al. Does physiological distribution of blood parameters in children depend on socioeconomic status? Results of a German cross-sectional study. BMJ Open. 2018;8(3):e019143.

14 Sun Y, Mensah FK, Azzopardi P, Patton GC, Wake M. Childhood social disadvantage and pubertal timing: a national birth cohort from Australia. Pediatrics. 2017;139(6):e20164099.

15 Deardorff J, Abrams B, Ekwaru JP, Rehkopf $\mathrm{DH}$. Socioeconomic status and age at menarche: an examination of multiple indicators in an ethnically diverse cohort. Ann Epidemiol. 2014;24(10):727-33.

16 James-Todd T, Tehranifar P, Rich-Edwards J, Titievsky L, Terry MB. The impact of socioeconomic status across early life on age at menarche among a racially diverse population of girls. Ann Epidemiol. 2010;20(11): $836-42$.

17 Zhang L, Zhang D, Sun Y. Adverse childhood experiences and early pubertal timing among girls: a meta-analysis. Int J Environ Res Public Health. 2019 Aug 13;16(16):2887.

18 Quante M, Hesse M, Döhnert M, Fuchs M, Hirsch C, Sergeyev E, et al. The LIFE Child study: A life course approach to disease and health. BMC Public Health. 2012;12:1021.

19 Marshall WA, Tanner JM. Variations in pattern of pubertal changes in girls. Arch Dis Child. 1969;44(235):291.

20 Marshall WA, Tanner JM. Variations in the pattern of pubertal changes in boys. Arch Dis Child. 1970;45(239):13-23.

21 Zachmann M, Prader A, Kind HP, Häfliger H, Budliger $\mathrm{H}$. Testicular volume during adolescence. Cross-sectional and longitudinal studies. Helv Paediatr Acta. 1974;29(1):61-72.

22 Kromeyer-Hauschild K, Wabitsch M, Kunze D, Geller F, Geiß HC, Hesse V, et al. Perzentile für den body-mass-index für das Kindes- und Jugendalter unter Heranziehung verschiedener deutscher Stichproben. Monatsschr Kinderheilkd. 2001;149(8):807-18.

23 Winkler J, Stolzenberg H. Adjustierung des Sozialen-Schicht-Index für die Anwendung im Kinder-und Jugendgesundheitssurvey (KiGGS). Berlin, Germany: Wismarer Diskussionspapiere; 2009.
24 Hiatt RA, Stewart SL, Hoeft KS, Kushi LH, Windham GC, Biro FM, et al. Childhood socioeconomic position and pubertal onset in a cohort of multiethnic girls: implications for breast cancer. Cancer Epidemiol Biomarkers Prev. 2017;26:1714-21.

25 Frisch RE. Body fat, menarche, fitness and fertility. Hum Reprod. 1987;2(6):521-33.

26 Heger S, Körner A, Meigen C, Gausche R, Keller A, Keller E, et al. Impact of weight status on the onset and parameters of puberty: analysis of three representative cohorts from central Europe. J Pediatr Endocrinol Metab. 2008;21(9):865.

27 Shalitin S, Kiess W. Putative effects of obesity on linear growth and puberty. Horm Res Paediatr. 2017;88(1):101-10.

28 Ahmed ML, Ong KK, Dunger DB. Childhood obesity and the timing of puberty. Trends Endocrinol Metab. 2009;20(5):237-42.

29 Wagner IV, Sabin M, Kiess W. Influences of childhood obesity on pubertal development. Metabolic syndrome and obesity in childhood and adolescence. Pediatr Adolesc Med Karger. 2015;19:110-25.

30 Aksglaede L, Juul A, Olsen LW, Sørensen TI. Age at puberty and the emerging obesity epidemic. PLoS One. 2009;4(12):e8450.

31 Juul A, Magnusdottir S, Scheike T, Prytz S, Skakkebaek NE. Age at voice break in Danish boys: effects of pre-pubertal body mass index and secular trend. Int J Androl. 2007;30(6): 537-42.

32 Kaplowitz PB, Slora EJ, Wasserman RC, Pedlow SE, Herman-Giddens ME. Earlier onset of puberty in girls: relation to increased body mass index and race. Pediatrics. 2001;108(2): 347-53.

33 Ohlsson C, Bygdell M, Celind J, Sondén A, Tidblad A, Sävendahl L, et al. Secular trends in pubertal growth acceleration in Swedish boys born from 1947 to 1996 . JAMA Pediatr. 2019;173(9):860-5.

34 Mouritsen A, Aksglaede L, Soerensen K, Hagen $\mathrm{CP}$, Petersen JH, Main KM, et al. The pubertal transition in 179 healthy Danish children: associations between pubarche, adrenarche, gonadarche, and body composition. Eur J Endocrinol. 2013;168(2):129-36.

35 Obeidallah DA, Brennan RT, Brooks-Gunn J, Kindlon D, Earls F. Socioeconomic status, race, and girls' pubertal maturation: results from the project on human development in Chicago neighborhoods. J Res Adolesc. 2000; 10:443-64.

36 Cohen S, Janicki-Deverts D, Chen E, Matthews KA. Childhood socioeconomic status and adult health. Ann N Y Acad Sci. 2010; 1186:37-55.
37 Belsky J, Steinberg L, Draper P. Childhood experience, interpersonal development, and reproductive strategy: an evolutionary theory of socialization. Child Dev. 1991;62:647-70.

38 Nottelmann ED, Susman EJ, Dorn LD, InoffGermain G, Loriaux DL, Cutler GB, et al. Developmental processes in early adolescence. Relations among chronologic age, pubertal stage, height, weight, and serum levels of gonadotropins, sex steroids, and adrenal androgens. J Adolesc Health Care. 1987;8(3):24660.

39 Vliegenthart J, Noppe G, van Rossum EF, Koper JW, Raat H, van den Akker EL. Socioeconomic status in children is associated with hair cortisol levels as a biological measure of chronic stress. Psychoneuroendocrinology. 2016;65:9-14.

40 Ruttle PL, Shirtcliff EA, Armstrong JM, Klein MH, Essex MJ. Neuroendocrine coupling across adolescence and the longitudinal influence of early life stress. Dev Psychobiol. 2015; 57(6):688-704.

41 Hochberg Z, Belsky J. Evo-devo of human adolescence: beyond disease models of early puberty. BMC Med. 2013;11:113-11.

42 Ellis BJ. Timing of pubertal maturation in girls: an integrated life history approach. Psychol Bull. 2004;130(6):920-58.

43 Biro FM, Lucky AW, Huster GA, Morrison JA. Pubertal staging in boys. J Pediatr. 1995; 127(1):100-2.

44 Sequera AM, Fideleff HL, Boquete HR, Pujol $A B$, Suárez MG, Ruibal GF. Basal ultrasensitive LH assay: a useful tool in the early diagnosis of male pubertal delay? J Pediatr Endocrinol Metab. 2002;15(5):589.

45 Surana V, Dabas A, Khadgawat R, Marwaha RK, Sreenivas V, Ganie MA, et al. Pubertal onset in apparently healthy Indian boys and impact of obesity. Indian J Endocrinol Metab. 2017;21(3):434-8.

46 Lee PA, Gollenberg AL, Hediger ML, Himes $\mathrm{JH}$, Zhang Z, Louis GMB. Luteinizing hormone, testosterone and inhibin $B$ levels in the peripubertal period and racial/ethnic differences among boys aged 6-11 years: analyses from NHANES III, 1988-1994. Clin Endocrinol. 2010;73:744-51.

47 Houk CP, Kunselman AR, Lee PA. Adequacy of a single unstimulated luteinizing hormone level to diagnose central precocious puberty in girls. Pediatrics. 2009;123(6):e1059-63.

48 Filc D, Davidovich N, Novack L, Balicer RD. Is socioeconomic status associated with utilization of health care services in a single-payer universal health care system? Int J Equity Health. 2014;13:115. 